Computing the correlation and other things directly from the raw pairs

David A. Brown

September 4, 2007 
This document was prepared as an account of work sponsored by an agency of the United States Government. Neither the United States Government nor the University of California nor any of their employees, makes any warranty, express or implied, or assumes any legal liability or responsibility for the accuracy, completeness, or usefulness of any information, apparatus, product, or process disclosed, or represents that its use would not infringe privately owned rights. Reference herein to any specific commercial product, process, or service by trade name, trademark, manufacturer, or otherwise, does not necessarily constitute or imply its endorsement, recommendation, or favoring by the United States Government or the University of California. The views and opinions of authors expressed herein do not necessarily state or reflect those of the United States Government or the University of California, and shall not be used for advertising or product endorsement purposes.

This work was performed under the auspices of the U.S. Department of Energy by University of California, Lawrence Livermore National Laboratory under Contract W-7405-Eng-48. 


\title{
Computing the correlation and other things directly from the raw pairs
}

\author{
D.A. Brown \\ Lawrence Livermore National Laboratory, Livermore California 94551
}

(Dated: August 16, 2007)

PACS numbers: PACS numbers: 25.75.-q, 25.75.Gz

We want a faster and more robust way to compute the correlation, expanded in Spherical (or Cartesian) Harmonics. We also want to include the cross- $\ell, m$ data covariance that are there, but currently ignored. We don't want to get bogged down in fancy binning in $x-y-z$ or $r-\theta-\phi$, just $r$. Want to just look at $C_{\ell m}$ to decide how many terms to keep - or better yet the pair distributions directly.

First, we need the true pair and mixed pair distributions $T(\mathbf{q})$ and $M(\mathbf{q})$ from which we could compute the correlation $C(\mathbf{q})$ :

$$
T(\mathbf{q})=C(\mathbf{q}) M(\mathbf{q})
$$

If we expand the pair distributions and the correlation in Spherical Harmonics (for the sake of argument), then we have

$$
\sqrt{4 \pi} \sum_{\ell m} T_{\ell m}(q) Y_{\ell m}\left(\Omega_{\hat{\mathbf{q}}}\right)=\left(\sqrt{4 \pi} \sum_{\ell^{\prime} m^{\prime}} M_{\ell^{\prime} m^{\prime}}(q) Y_{\ell^{\prime} m^{\prime}}\left(\Omega_{\hat{\mathbf{q}}}\right)\right)\left(\sqrt{4 \pi} \sum_{\ell^{\prime \prime} m^{\prime \prime}} C_{\ell^{\prime \prime} m^{\prime \prime}}(q) Y_{\ell^{\prime \prime} m^{\prime \prime}}\left(\Omega_{\hat{\mathbf{q}}}\right)\right)
$$

So,

$$
\begin{aligned}
T_{\ell m}(q) & =\sqrt{4 \pi} \sum_{\ell^{\prime} m^{\prime} \ell^{\prime \prime} m^{\prime \prime}} M_{\ell^{\prime} m^{\prime}}(q) C_{\ell^{\prime \prime} m^{\prime \prime}}(q) \int_{4 \pi} d \Omega_{\hat{\mathbf{q}}} Y_{\ell m}^{*}\left(\Omega_{\hat{\mathbf{q}}}\right) Y_{\ell^{\prime} m^{\prime}}\left(\Omega_{\hat{\mathbf{q}}}\right) Y_{\ell^{\prime \prime} m^{\prime \prime}}\left(\Omega_{\hat{\mathbf{q}}}\right) \\
& =\sqrt{4 \pi} \sum_{\ell^{\prime} m^{\prime} \ell^{\prime \prime} m^{\prime \prime}} M_{\ell^{\prime} m^{\prime}}(q) C_{\ell^{\prime \prime} m^{\prime \prime}}(q)(-1)^{m} \int_{4 \pi} d \Omega_{\hat{\mathbf{q}}} Y_{\ell m}\left(\Omega_{\hat{\mathbf{q}}}\right) Y_{\ell^{\prime} m^{\prime}}\left(\Omega_{\hat{\mathbf{q}}}\right) Y_{\ell^{\prime \prime} m^{\prime \prime}}\left(\Omega_{\hat{\mathbf{q}}}\right) \\
& =\sqrt{4 \pi} \sum_{\ell^{\prime} m^{\prime} \ell^{\prime \prime} m^{\prime \prime}} M_{\ell^{\prime} m^{\prime}}(q) C_{\ell^{\prime \prime} m^{\prime \prime}}(q)(-1)^{m}\left[\frac{(2 \ell+1)\left(2 \ell^{\prime}+1\right)\left(2 \ell^{\prime \prime}+1\right)}{4 \pi}\right]^{1 / 2}\left(\begin{array}{cccc}
\ell & \ell^{\prime} & \ell^{\prime \prime} \\
0 & 0 & 0
\end{array}\right)\left(\begin{array}{ccc}
\ell & \ell^{\prime} & \ell^{\prime \prime} \\
-m & m^{\prime} & m^{\prime \prime}
\end{array}\right)(5) \\
& =\sum_{\ell^{\prime} m^{\prime} \ell^{\prime \prime} m^{\prime \prime}} M_{\ell^{\prime} m^{\prime}}(q) C_{\ell^{\prime \prime} m^{\prime \prime}}(q)(-1)^{m} \sqrt{(2 \ell+1)\left(2 \ell^{\prime}+1\right)\left(2 \ell^{\prime \prime}+1\right)}\left(\begin{array}{ccccc}
\ell & \ell^{\prime} & \ell^{\prime \prime} \\
0 & 0 & 0
\end{array}\right)\left(\begin{array}{ccc}
\ell & \ell^{\prime} & \ell^{\prime \prime} \\
-m & m^{\prime} & m^{\prime \prime}
\end{array}\right) \\
& \equiv \sum_{\ell^{\prime \prime} m^{\prime \prime}} \tilde{M}_{\ell m \ell^{\prime \prime} m^{\prime \prime}}(q) C_{\ell^{\prime \prime} m^{\prime \prime}}(q)
\end{aligned}
$$

Eq. (7) gives us a was to compute $C_{\ell m}(q)$ directly from the pair distributions expanded in spherical harmonics. All we need to do is invert the matrix in $\ell m \ell^{\prime \prime} m^{\prime \prime}$; Eq. (7) is an imaging problem where $\tilde{M}$ is the kernel, $T$ is the data and $C$ is the model we seek to reconstruct. We can even do error propagation in the obvious way, provided we measure the mixed pair distribution so well that the uncertainties are negligible compared to the true distribution. What's more, when you do the error propagation in the obvious way, you get the cross- $\ell m$ correlations in the covariance matrix by virtue of the cross- $\ell m$ correlations built into the $\tilde{M}$ matrix. All of this can be done with the current imaging code inside of CorAL, provided the appropriate wrappers are written. Also, it is possible to include the covariance in the mixed pair distribution too. This would require writing a new imaging core routine which allows for uncertainties on the kernel.

Now, how to we compute $T_{\ell m}(q)$ (or $\left.M_{\ell^{\prime} m^{\prime}}(q)\right)$ ? First, we return to the spherical harmonic expansion of the pair distribution:

$$
T(\mathbf{q})=\sqrt{4 \pi} \sum_{\ell m} T_{\ell m}(q) Y_{\ell m}\left(\Omega_{\hat{\mathbf{q}}}\right)
$$

so

$$
T_{\ell m}(q)=\int_{4 \pi} d \Omega_{\hat{\mathbf{q}}} T(\mathbf{q}) Y_{\ell m}^{*}\left(\Omega_{\hat{\mathbf{q}}}\right)
$$

We build this up by summing over pairs, which is essentially a Monte-Carlo integration process:

$$
T_{\ell m}\left(q_{n}\right) \approx\left\{\begin{aligned}
\frac{1}{N} \sum_{i=1}^{N} Y_{\ell m}^{*}\left(\Omega_{\hat{\mathbf{q}}_{i}}\right) & \text { if } q_{i} \text { in bin } n \\
0 & \text { otherwise }
\end{aligned}\right.
$$


with covariance (for if $q_{i}$ in bin $n$ only:)

\section{Acknowledgements}

$\Delta^{2} T_{\ell m \ell^{\prime} m^{\prime}}\left(q_{n}\right) \approx \frac{1}{N-1}\left(\frac{1}{N} \sum_{i=1}^{N} Y_{\ell m}^{*}\left(\hat{\mathbf{q}}_{i}\right) Y_{\ell^{\prime} m^{\prime}}^{*}\left(\hat{\mathbf{q}}_{i}\right)-T_{\ell m}\left(q_{n}\right) T_{\ell^{\prime} m^{\prime}}\left(q_{n}\right)\right)$

giving (uncorrelated) uncertainties of

(11) This work was performed under the auspices of the U.S. Department of Energy by Lawrence Livermore Na-

$$
\Delta T_{\ell m}\left(q_{n}\right) \approx \sqrt{\Delta^{2} T_{\ell m \ell m}\left(q_{n}\right)}
$$
tional Laboratory under Contract W-7405-Eng-48.

(Note to reader: check those $1 / \mathrm{N}$ type factors. I couldn't find a statistics book to check them) 